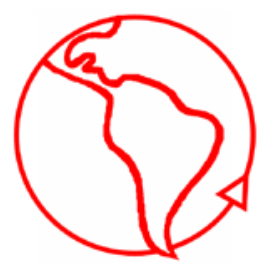

\section{Los misterios del dinero}

\section{The Mysteries of Money}

\section{Elena Esposito}

Departamento de Comunicación y Economía, Universidad de Módena y Reggio Emilia, Italia.

Resumen

El presente artículo aborda los problemas relativos a la comprensión del dinero en la sociedad moderna. En la actualidad no existe una teoría sociológica adecuada para comprender las funciones y especificidades del dinero. Frente a esto el presente artículo se propone comprender al dinero en el contexto de una dimensión temporal, específicamente como un "medio de aplazamiento". Se abordan a continuación algunas consecuencias de la monetarización de la economía de la sociedad y se analizan algunas características de la economía de los "derivados". El artículo concluye con consideraciones relativas al nuevo carácter de la economía monetaria.

Palabras Clave: Dinero; Tiempo; Economía, Monetarización; Derivados

\begin{abstract}
This article addresses problems of the understanding of money in modern society. At present there is no adequate sociological theory to understand functions and specificities of money. Faced with this, the present article aims to define money in the context of a temporal dimension, specifically as a "medium of deferment". Some consequences of the monetarization of the economy of society and some characteristics of the economy of the "derivatives" are addressed. The article concludes with considerations concerning the new character of the monetary economy.
\end{abstract}

Keywords: Money; Time; Economy; Monetarization; Derivatives

\title{
Introducción*
}

El dinero siempre ha despertado desconfianza y sospecha. La famosa definición del dinero de Lutero (1883: 391) como "excremento del diablo" es parte de una larga tradición que asocia el dinero con el demonio. Esto se debe a que el dinero resulta incomprensible y amenaza tanto al orden establecido como a la armonía de las cosas y del mundo. En la Edad Media se daba por sentado que el dinero era "una cábala difícil de entender" (Braudel 1967: 365), no solo porque este era técnico, y por lo tanto complicado, sino también porque era fuente de absurdas paradojas -por ejemplo, el dinero que se presta regresa a su fuente en la misma cantidad, multiplicando la masa de dinero circulante. Esto ocurre de manera tal que la cantidad de dinero resulta ser la misma y al mismo tiempo diferente. Acertijos de este tipo se pueden encontrar por doquier y hasta el siglo XVII no hubo disponible una verdadera teoría del dinero. En el siglo XVII se hizo evidente, sin embargo, el contraste entre el tradicional desprecio por el dinero y el nuevo descubrimiento que "todo se puede comprar con dinero" (Rice Vaughn 1675, citado en Appleby 1978: 199).

\footnotetext{
* [N. del T.] Traducción de Hugo Cadenas. Título original: The Mysteries of Money. Inaesthetics, Núm. 3, 2012, pp. 21-28. Publicado con autorización de Elena Esposito. El texto ha sido revisado y editado especialmente para este número de la revista gracias a la gentil colaboración de la autora.
} 
Este instrumento, el dinero, suscitó tanto entusiasmo como inquietud debido a que, a través de la atribución de un valor, parecía capaz de poner en condiciones de igualdad cosas tan disímiles como un día de trabajo, una carga de telas exóticas, los bienes familiares y el costo de un préstamo, y permitía la comparación entre todo ello, disolviendo definitivamente muchas de las estructuras sociales tradicionales (Luhmann 1988a: 230 y ss. $)^{1}$.

El funcionamiento del dinero se ha vuelto hoy en día aún más improbable: el dinero no solamente homogeneíza todos los bienes y valores, haciéndolos comparables, sino parece ser capaz también de nivelar todos los riesgos y todas las relaciones con el futuro. La economía ofrece la posibilidad de asegurarse (pagar) frente a la preocupante perspectiva de un futuro daño (de cualquier fuente). Uno puede contratar un seguro, el cual (como dice el propio término) compensa el riesgo con seguridad, aunque sea solo mediante una seguridad económica. Si uno está preocupado de enfermarse, sufrir un accidente o dañar a otras personas, el seguro no garantiza que el temido evento no ocurra, sino solo que uno recibirá una suma de dinero en caso que suceda. El dinero no posee ningún uso intrínseco, sin embargo, posee la extraña característica de ofrecer garantías contra la incertidumbre para todas y cada una de las personas. Ya sea de metal o de papel, el dinero no tiene ninguna utilidad. Entonces, ¿por qué ha llegado a ser tan útil?

Incluso hoy en día no existe una teoría que pueda explicar de manera convincente el funcionamiento del dinero y sus condiciones. Los propios economistas consideran que es necesaria una teoría real del dinero. La teoría económica neoclásica, sostienen, se basa implícitamente en el modelo del trueque (cf. por ejemplo Hicks 1967; Aglietta \& Orléan 1982: 13; Robinson 1971). Este modelo se define, de hecho, como la teoría de un sistema que funciona sin dinero, donde los bienes son lo que realmente importa, y el dinero es solo una abstracción que se produce a posteriori. En dicho modelo se piensa en un sistema "real" (el mercado) que opera mediante la distribución de bienes, con el "velo del dinero" puesto en la parte superior de él. Los economistas deberían romper ese velo y mudarse desde el plano "metafísico" de las entidades ligeras hacia el plano genuino de las fuerzas que producen la riqueza ${ }^{2}$. Desde el punto de vista previo, el dinero emerge como consecuencia del mercado y no tiene ningún poder en sí mismo. No puede crear una riqueza real, solo una riqueza ilusoria. Según los críticos, este enfoque pasa por alto por completo el poder real y la verdadera naturaleza del dinero. El dinero no es pues una entidad metafísica, sino un hecho muy real y concreto. No es que el dinero emerja del mercado, sino que el mercado y su poder son consecuencia de la monetarización de la economía (cf. Ingham 2000: 17). El dinero puede ser abstracto, pero es una "abstracción real"3, y hay que explicarlo como tal.

\section{El tiempo del dinero y las necesidades}

La debilidad de la economía se constata en su ambigua definición de la función del dinero y en la distinción estándar respecto de las tres tareas que este realiza: medida de los valores, medio de pago y medio de intercambio ${ }^{4}$. En efecto, la relación entre estas tres funciones es muy vaga. Resulta difícil entender cuál ocurrió primero y si es posible derivar una de las otras. ¿Existe una función primaria? ¿Cuál es? ¿Cómo afecta a las otras tareas del dinero?

\footnotetext{
${ }^{1}$ Luhmann habla aquí del dinero como un medio simbólico y diabólico al mismo tiempo.

${ }^{2}$ La crítica a este modelo se puede encontrar ya en Keynes (1936: 18 y ss.). Cf. también Robinson (1971: 64 y ss.) y Smithin (2000). Para una discusión sobre la ilusión de neutralidad del dinero, véase Ingham (2004: 15 y ss.).

${ }^{3}$ De acuerdo con la expresión de Sohn-Rethel (1990).

${ }^{4}$ Cf. por ejemplo, Hicks (1967), Bloch (1954: 35), pero también Polany (1957: 296-331, trad. it.), Ingham (2004). Weber (1922: 70 y ss., trad. it.) define al dinero sobre la base de su naturaleza dual como medio de intercambio y medio de pago, lo que conduce a la posibilidad de que este sirva de referencia para ser utilizado en futuras oportunidades aún indeterminadas.
} 
La gran ventaja del dinero es que deja completamente abierto el momento en que uno debe gastarlo. Debido a que es abstracto e indeterminado su valor se mantiene, incluso si uno aguarda antes de gastarlo. Este queda disponible incluso cuando se pospone la decisión en vista de otras situaciones, de otros socios o de diferentes condiciones. El significado y la función del dinero estriba en su dilación temporal, en la posibilidad que ofrece para usar el tiempo de modo de aumentar las opciones de elección y decisión ${ }^{5}$. Esta era por ejemplo la opinión de Keynes (1936: 293). Él sostuvo explícitamente que la importancia del dinero reside en que este es "un vínculo entre el presente y el futuro" que se hace necesario cuando el futuro es desconocido y por lo tanto es "pérfido" y amenazador.

Esta función del dinero resulta necesaria solamente en una economía moderna, la cual (al igual que cualquier otro segmento de la sociedad) se enfrenta a un futuro incierto e indeterminado, a sabiendas que depende de decisiones en el presente. El futuro es por tanto aterrador, pero puede sin embargo $-y$, de hecho, debe- continuar. No sabemos qué va a pasar en el futuro. Sin embargo, a pesar de que nuestras expectativas pueden estar erradas, el valor del dinero se mantiene y puede ser utilizado en condiciones diferentes a las que inicialmente esperábamos. El dinero nos posibilita actuar incluso si no podemos controlar las consecuencias, pues nos permite aplazar decisiones o acciones reteniendo su valor. No tenemos que decidir hoy cómo gastaremos el dinero y podemos operar como si tuviéramos dinero en el futuro con el fin de poder decidir más tarde. Solo en un mundo de incertidumbre el dinero puede tener esta función, ya que actúa como un "puente" entre los planes (usualmente equivocados) del pasado, las expectativas actuales y las expectativas frente a un futuro enigmático (cf. Davidson 1978: 146; Goodhart 1994: 55 y ss., trad. it.; Moore 1979: 123 y ss.).

Shackle (1990: 213; 1972: 160) lo ha señalado explícitamente. El dinero no es primariamente un depósito de valor, pues el valor cambia y se transforma. Tampoco es un medio de intercambio, pues no sabemos qué intercambiar o dónde obtenerlo. Es, en cambio, un "medio de aplazamiento y de búsqueda". Esta es su función principal, este rendimiento es el punto de partida para explicar su funcionamiento. Necesitamos dinero en un mundo que está orientado hacia un futuro incierto, debido a que el sentido de la monetarización descansa en la posibilidad de posponer la elección para otro día en el futuro, cuando uno tenga más información. La ventaja del dinero es que nos permite operacionalizar nuestra falta de conocimiento. Esto es posible porque el dinero, al no tener valor por sí mismo, permanece totalmente indeterminado. No es vinculante para decisiones futuras, aparte del simple hecho que uno eventualmente va a tener que tomar una decisión -esto es, gastar. En el trueque la ignorancia nos pone en desventaja, porque uno no sabe si y cuándo va a encontrar los productos que a uno le interesan. Sin embargo, en una economía monetarizada, no se produce este efecto pues el dinero permite emplear tiempo para buscar información adicional respecto de cómo obtener estos bienes. El dinero, en otras palabras, es una herramienta que aborda la incertidumbre del futuro en el presente.

Esta protección contra un futuro amenazante (la cual es evidente en el caso de los seguros) es en efecto el rasgo básico de todas las operaciones económicas y constituye la función real de la economía. Si bien es cierto que los pagos siempre están destinados a satisfacer necesidades y estas son, por lo tanto, generalmente consideradas como la función principal de la economía ${ }^{6}$, un examen a fondo del concepto de necesidad parece

\footnotetext{
${ }^{5}$ El interés, una entidad a menudo misteriosa, se hace así comprensible: el interés mide y revela la relevancia temporal del dinero.

${ }^{6}$ También desde una perspectiva sociológica: baste pensar en Parsons y en la ubicación del subsistema económico en el cuadro del AGIL dedicado a la adaptación, es decir, a la adquisición de energía para satisfacer necesidades. Cf. también Weber (1922).
} 
ser extremadamente complicado y el concepto está tan fuertemente influenciado por la propia economía que su función se vuelve inverosímil. Se convierte en una demanda generada por la propia economía y que esta tiene que satisfacer (cf. Luhmann 1988a: 59 y S.; 1992: 39). ¿Cuáles son las necesidades reales y cómo pueden determinarse? ¿Dónde debe dibujarse la línea de demarcación entre las necesidades básicas y las necesidades creadas, entre las necesidades de lujo y las necesidades de aparentar?

Si las necesidades fueran un hecho antropológico o un requerimiento elemental estarían limitadas a los estratos más bajos de la sociedad y, por lo tanto, serían satisfechas de manera más o menos rápida (no tenemos muchas necesidades básicas). Sin embargo, las necesidades nunca se agotan y por lo tanto no desaparecen. Desde dicha perspectiva no se logra explicar la propagación de necesidades que no excluyen a ningún miembro de la sociedad. Ni siquiera se puede explicar el hecho que nunca hay suficiente dinero, por ricos que seamos, pues no existen límites para la abstracta urgencia de necesidades. Esto se debe al dinero en sí mismo y no a las necesidades. El dinero que uno puede gastar como crea conveniente (incluso de maneras que uno todavía no conoce) representa en su abstracción la generalidad de todas las necesidades posibles. Necesitamos dinero ahora porque no sabemos lo que podemos necesitar en el futuro, y estamos por tanto siempre necesitados, no de mercancías, sino de dinero. En lugar de satisfacer necesidades, la economía monetaria parece generar las necesidades sobre las cuales opera. Sin dinero no habrían necesidades que puedan ser satisfechas por el dinero.

Si las necesidades nunca se agotan y afectan a todos los miembros de la sociedad, entonces posiblemente no tienen que ver con lo que necesitamos hoy, sino que se dirigen al horizonte indeterminado donde la incertidumbre nunca es controlada, al futuro abierto e impredecible donde nunca se sabe qué necesidades pueden surgir. No podemos saber lo que vamos a necesitar, por lo que necesitamos más y más dinero con el fin de hacer frente a esta incertidumbre. La referencia del dinero se vuelca así hacia la incertidumbre del futuro y no hacia las necesidades en sí mismas. Esto se transforma en la verdadera referencia de la economía. La economía, debemos señalar, no se ocupa de las necesidades materiales, sino del tiempo, del tiempo abierto, incierto y recurrente que aparece como riesgo y que tiende a fundirse en la economía. Eric Voegelin (1925: 204) sostuvo esto hace casi un siglo: "El tiempo es el sentido de la economía". El punto es comprender cómo la economía produce y maneja el tiempo.

\section{El dinero como medio de aplazamiento y sus derivados}

¿Cómo puede este ente abstracto sin valor en sí mismo conducir la masa de bienes y valores que constituyen la muy concreta riqueza de la sociedad moderna? ¿Cómo puede motivar a todos sin tener un objetivo específico? No sabemos lo que en este minuto queremos, o lo que otros quieren, o lo que podríamos querer en el futuro, pero todos quieren dinero ${ }^{7}$. Rendimientos muy disímiles están todos compensados de la misma manera: con pagos.

La sociología responde siempre a este problema de la misma manera. El dinero funciona porque representa una relación social o porque es una relación social que consiste en obligaciones y exigencias entre los participantes en la economía ${ }^{8}$. De este modo argumentó Weber (1922, vol. II: 314, trad. it.), quien escribió que un intercambio que hace uso del dinero es siempre "acción comunitaria", que refiere implícitamente a la acción potencial de otros y, finalmente, a todos los participantes. El dinero solo se acepta porque se espera utilizarlo en futuras transacciones. Esto no solo depende de la contraparte actual, sino también de todos los potenciales otros que podrían estar interesados en el

\footnotetext{
${ }^{7}$ Hasta el punto que, como señaló Luhmann de una mera algo provocadora en referencia a las reivindicaciones de los sindicatos, el "optativo categórico" de la sociedad moderna es "imás dinero!".

${ }^{8}$ Una discusión actualizada sobre esto se puede encontrar en Ingham (2004).
} 
intercambio, de quienes también se espera que acepten dinero. Cada transacción monetaria, aunque se trate de una muy puntual y concreta, entre gente que se conoce y que puede confiar entre sí, se extiende más allá de este nivel y refiere a toda la comunidad, a todas las posibles transacciones anónimas e indeterminadas, entre personas desconocidas y en momentos que aún se desconocen. Como señala Simmel (1889: 49, trad. it.; 1900: 219 y 338 y s., trad. it.), el dinero puede cumplir esta función simbólica y conectar la peculiaridad de cada intercambio con la totalidad de los bienes y las personas, puesto que está desprovisto totalmente de un valor propio. Como la historia del dinero ha mostrado, cada remanente de un valor intrínseco del dinero se convierte en un obstáculo para su función primaria. Su valor radica solamente en su capacidad para convertirse en otros valores. Su uso como un medio radica únicamente en su capacidad para convertirse en un fin. Como un símbolo para cada valor, esto significa que la ausencia de valor se convierte en el último valor y el último objetivo por el cual todos se afanan. La confianza concreta en personas y relaciones entre diferentes individuos se convierte en una abstracta "confianza sistémica" en el funcionamiento de la economía en su totalidad.

El dinero puede motivar a cualquier persona, incluso a hacer cosas que de otro modo parecerían irracionales (como lo demuestra el amplio deseo de tener un trabajo -es decir, de trabajar). En esta función social de "homogeneización", en la cual se combinan sin conflictos una variedad de motivaciones individuales muy heterogéneas, el dinero parece asumir el rol antes desempeñado por la motivación religiosa, la cual también era válida para todas las personas. La motivación monetaria puede ser vista, según Burke (1969: 92 y ss., 110 y ss.), como "un sustituto técnico de Dios", pues Dios era la instancia unitaria que podría recoger la diversidad de todos los motivos. En este sentido, el dinero (según Simmel, entre otros) puede ser visto como "el Dios de nuestro tiempo" (Simmel 1889: 65, trad. it.), un Dios secular y socializado que abarca motivos y objetos indistintamente dispares.

Si el dinero ha sido siempre misterioso, hoy en día este misterio ha adquirido otra dimensión, emergiendo nuevos enigmas que aún permanecen oscuros. En las últimas décadas la economía ha cambiado profundamente, sobre todo debido a la mayor relevancia de las finanzas y por la introducción de instrumentos abstractos y transparentes como los derivados financieros. Hay quienes sugieren que los consideremos como una nueva forma de dinero, el cual sería propio del capitalismo financiero y sus movimientos, sería muy abstracto y conectado con la disponibilidad de computadoras (cf. Rotman 1987; Pryke \& Allen 2000; Bryan \& Rafferty 2007; Li Puma \& Lee 2005). Sin embargo, ¿por qué debería ser un nuevo tipo de dinero? ¿Cómo se superponen estas herramientas a la función y el modo de operación del dinero, el cual ha dado pie a la economía moderna autorreferencial y orientada hacia el futuro?

Como hemos visto, el dinero funciona sobre la base de su peculiar capacidad para la homogeneización. La monetarización convierte a la propiedad de bienes muy diversos (muebles, joyas, alimentos, así como la tierra y el trabajo) en equivalentes, al convertir dicha propiedad en una cantidad (una suma de dinero) que puede ser comparada e intercambiada con cualquier otra suma de dinero. Desde el punto de vista del capital que representan, todos los bienes son equivalentes $y$, por lo tanto, homogéneos. El dinero puede enlazar con el futuro, ya que hace equivalente a cualquier propiedad frente a otra. De este modo, permite la disponibilidad (o transferencia) de bienes presentes equivalentes a los bienes indeterminados que serían accesibles en el futuro. Atribuimos la función primaria del dinero a este rendimiento, a su capacidad de actuar como medio de aplazamiento. Sus funciones clásicas como medio de cambio y provisión de valor son consecuencia de este rendimiento. Si los derivados antes mencionados constituyen una clase particular de dinero, ¿hacen también ellos algo similar? ¿Cómo?

Bryan y Rafferty (2007: 140 y s.) sostienen que estos derivados son una nueva forma de dinero, ya que poseen la capacidad específica de enlazar con el futuro apoyándose en una 
peculiar forma de homogeneización (combinación); una homogeneización de segundo nivel que presupone la abstracción de la economía moderna y la transformación de bienes en capital. Los derivados son capaces de equiparar diferentes formas de capital (y no diferentes formas de bienes) y se pueden transformar (cf. Li Puma \& Lee 2005: 411). En la economía anterior, se distingue entre diferentes tipos de capital: capital de producción, capital comercial (que relaciona oferta y demanda), capital de crédito, generados todos por la circulación del capital mismo. En el tráfico de derivados, todas las formas de capital se convierten en equivalentes, debido a que pueden convertirse unas en otras con el fin de generar la liquidez que circula en el mercado.

¿Cuál es el mecanismo que permite a los derivados adquirir este grado de libertad? En el tráfico de derivados, la dinámica de las inversiones es independiente de la propiedad. Se puede invertir en un título, influyendo así en sus movimientos y se pueden lograr beneficios o pérdidas sin necesidad de poseer dicho título. Al igual que en las ventas en corto, donde uno vende algo que no posee, para los derivados es suficiente con ser capaz de gestionar determinadas transacciones, especulando sobre tendencias y expectativas. Cuando se trata de los futuros, se invierte en los movimientos futuros de un activo que no se posee (y que con frecuencia nunca se poseerá, ya que el futuro será vendido antes que expire). Las opciones otorgan incluso la libertad de no tener que poseer nada. Las permutas de incumplimiento crediticio (credit default swap o CDS en inglés) entre los instrumentos financieros más innovadores, permiten ingresar en una especie de seguro sin necesidad que el comprador realmente posea los activos que desea proteger, o incluso pueda sufrir realmente una pérdida. Es como si uno estuviera asegurado contra el incendio de una casa que no se posee, o ante el riesgo de accidentes del automóvil de otra persona. Si se produce el daño, entonces se obtiene la compensación sin sufrir el daño. Esta independencia del dinero respecto de la propiedad es una novedad fundamental y representa un cambio decisivo en las operaciones de las finanzas y en la economía en su totalidad.

La referencia a la propiedad es, por supuesto, el centro de la dinámica económica y constituye el vínculo con el "mundo real". La monetarización dio lugar a la economía moderna con sus dinámicas autónomas guiadas por sus propios criterios, cuando el dinero realizó una "segunda codificación" de la propiedad, haciendo vinculante al tiempo correspondiente de manera mucho más flexible y fungible (cf. Luhmann 1988: 197 y ss.). La primera forma de protección frente a la incertidumbre del futuro fue suministrada por la propiedad, dando al titular la posibilidad de disfrutar de los bienes, incluso si otros aspiraban a ellos. Cuando los bienes son escasos y resultan de interés para muchos, el propietario tiene la facultad reconocida de proveerlos como estime conveniente, incluso si lo hace muy pobremente (desperdiciándolos o subutilizándolos de manera inadecuada). Si algo me pertenece a mí, soy libre de estropearlo o hacer que vaya mal, incluso si otros lo necesitan. La sociedad (es decir, los otros) me garantiza esta condición (una combinación sorprendentemente improbable).

La monetarización hace a este mecanismo mucho más eficiente. Inicia la dinámica autónoma que define a la economía y la hace en gran medida independiente de las características específicas de los bienes. La propiedad permanece como el requisito previo, con las (improbables) formas de apoyo social en las que se basa. Sin embargo, es traducida a una medida abstracta (el precio) que circula de forma independiente y que no está constreñida por la especificidad del contexto. Si mi propiedad ha sido pagada en 1.000 , puedo gastar esos 1.000 para obtener cualquier otro bien de características no especificadas, sin preocuparme del hecho que la transacción original tenía que ver con manzanas, casas o ganado. El dinero siempre presupone propiedad, pero convierte a la propiedad en abstracta e independiente de la especificidad del objeto (puedo cambiarla por cualquier otro bien), de la relación social con otras partes (puedo pagar a cualquiera), y del momento de la transacción (puede ocurrir en cualquier momento). 
Los derivados parecen liberar la relación entre el dinero y la propiedad, permitiendo comprar y vender seguridades que no se poseen. En los mercados financieros solo el dinero circula, sin ningún tipo de acoplamiento con la propiedad. ¿Sigue siendo esto dinero? ¿Cómo? Se puede sostener que se trata de una nueva forma de dinero que supone la abstracción alcanzada por la monetarización, pero yendo un paso más allá. Se abstrae de la especificidad del capital, haciendo a cada forma de capital convertible en cualquier otra y, finalmente, haciendo equivalente al dinero en sí mismo. Liberada de la propiedad, la circulación de las inversiones (el "nuevo" dinero) se convierte en liquidez pura que se comporta libremente, convirtiendo al dinero en dinero -es decir, comprando y vendiendo dinero a fin de comprar y vender (solo) más dinero. Al igual que la "primera" monetarización, la cual logró la independencia de las características de los bienes (de nuevo, una suma de dinero es la misma ya sea que se hayan vendido caballos o trigo), la "segunda" monetarización logró también la independencia de las características del capital. En los mercados de derivados se pueden obtener ganancias, incluso si los "subyacentes" van mal, del mismo modo que se pueden obtener pérdidas, incluso si la acción va en aumento. Uno no depende de la inversión. Uno no es dueño, ni siquiera parcialmente como en el caso de las acciones. En lugar de ello, uno depende de la observación de las inversiones en el mercado -es decir, de la velocidad y la intensidad de los movimientos de capital, sea lo que fuere a lo que se refieran.

La función más evidente de los derivados (reconocida también por sus opositores) es la creación de liquidez, aumentando ostensiblemente la masa de dinero circulante. Cada tipo de capital puede ser utilizado como "subyacente" a fin de generar un tráfico adicional de divisas, para entrar en nuevas inversiones, o para a retirarse e invertir en otra ocasión. Los mercados se vuelven más móviles, flexibles y dinámicos. Este dinero que circula en cantidades exorbitantes a velocidades muy altas y de manera aparentemente ilimitada es diferente, sin embargo, del dinero que hemos conocido desde hace siglos, el cual es la base de la monetarización moderna. Es diferente porque puede incrementarse de manera indeterminada. No tiene necesidad de permanecer acoplado a la propiedad de los bienes y activos. El dinero expresado por los derivados es también diferente debido a su función de segundo nivel de "combinación". No solo homogeniza las variadas formas del capital, sino que también borra la distinción entre el capital y el dinero. En definitiva, todo se convierte en líquido. El dinero en sí asume las características del capital (por ejemplo, puede generar más dinero $)^{9}$.

\section{Conclusión}

Como señaló Myron Scholes en su discurso de aceptación del premio Nobel (1997), refiriéndose a la confusión progresiva respecto de la distinción entre deudas y activos, todos los capitales pueden ser traducidos inmediatamente en dinero, aun cuando se trata de capital negativo (es decir, una deuda). Incluso una deuda puede convertirse en la base para la creación de riqueza, como es posible de apreciar en el uso de la pirámide de deudas de los bancos. Los bancos prestan dinero y tradicionalmente se pensaba que este dinero era depositado por los clientes. Hoy en día, sin embargo, las garantías puestas en la base de la circulación del capital se han convertido cada vez más en las deudas de aquellos que contratan préstamos o hipotecas, las cuales desembocan en otros bancos y se transforman en dinero en efectivo. En prácticas como la titulización, uno cede actividades o bienes a través de la emisión y colocación de bonos.

Estos pueden ser bienes raíces, contratos u otros, sin embargo los bienes transferidos son cada vez y más a menudo créditos (deudas de los clientes), transferidos a otros mediante pagos en efectivo. La maniobra tiene poco que ver con los bienes en concreto (propiedades) en la base de la pirámide (como casas o automóviles que a menudo son

\footnotetext{
${ }^{9}$ Esta es la tesis de Bryan \& Rafferty (2007: 153).
} 
objeto de otros préstamos), sino que descansa en un uso peligroso y temerario del tiempo. Los bienes son siempre los mismos, pero los diversos pagos (aquellos de los prestatarios, también los del primer banco y luego los de todos los demás operadores financieros) se superponen entre sí -es decir, son diversos los aplazamientos, diversos espacios de tiempo que se superponen entre sí. Dicha maniobra, como han mostrado en repetidas ocasiones las crisis o los estallidos de burbujas especulativas, no funciona cuando todos estos tiempos se acumulan unos sobre otros. Allí todos quieren sacar provecho simultáneamente y el juego de los aplazamientos colapsa sobre sí mismo.

Al igual que el dinero tradicional que se basa en la homogeneización de los bienes para enlazar con el futuro, los derivados realizan el rendimiento vertiginoso de creación del futuro y alcanzan un segundo nivel de homogeneización para enlazar con el tiempo de una manera novedosa. De este modo, pueden ser considerados una nueva forma de dinero. En este caso, sin embargo, el presupuesto es un nuevo paso de abstracción que permite una libertad de movimiento antes inconcebible -una "desmaterialización" que conduce a la reproducción financiera de cualquier inversión, sin necesidad de contar con el dinero para hacerla. En este nivel, ya no tiene mucho sentido mantener la distinción entre economía real y economía de papel, ya que todo se homogeneíza en un flujo indiferenciado de dinero abstracto.

Los derivados son una forma altamente autorreferencial del dinero, en el sentido que no se refieren a nada externo, sino solo al dinero y su circulación. En los mercados financieros, el dinero permanece para generar más dinero. Su valor es creado, no en referencia al mundo, sino en referencia al futuro. En su nueva forma financiera, se hace aún más evidente que el dinero es tiempo y, en efecto, el círculo de los derivados, sea este vicioso o virtuoso, solo tiene sentido si el tiempo entra en juego desplegando la tautología en una orientación hacia el futuro. En los términos de Rotman (1987: 153, trad. alem.), un derivado es una forma de dinero (Xenomoney) que crea su referencia por sí misma, "una señal que se crea a sí misma en el futuro". El valor es creado en el cálculo presente de los rendimientos futuros, los cuales a su vez forman parte del presente. RM

\section{Bibliografía}

Aglietta, M \& Orlean, A. (1982). La Violence de la monnaie. Paris: P.U.F.

Appleby, J. (1978). Economic Thought and Ideology in Seventeenth-Century England. Princeton, NJ: Princeton University Press.

Bloch, M. (1981). Lineamenti di una storia monetaria d'Europa. Torino: Einaudi.

Braudel, F. (1967). Civilisation matérielle, économie et capitalisme, XV'-XVIII siècle. Paris: Armand Colin. [Braudel, F. (1977). Capitalism a e civita materiale (secoli XV-XVIII). Torino: Einaudi].

Bryan, D. \& Rafferty, M. (1967). Financial Derivatives and the Theory of Money. Economy and Society, 36(1), 134- 158.

Burke, K. (1969). A Grammar of Motives. Berkeley, L.A./ London: University of California Press.

Davidson, P. (1972). Money and the Real World. London: MacMillan.

Goodhart, C. (1989). Money, Information and Uncertainty. London: MacMillan. [Goodhart, C. (1994). Moneta, Informazione e incertezza. Bologna: II Mulino]

Hicks, J. (1967). Critical Essays in Monetary Theory. Oxford: Clarendon Press.

Ingham, G. (2000). 'Babylonian madness'. On the historical and sociological origins of money. En J. Smithin (Ed.), What is Money? (pp. 16-41). London: Routledge.

Ingham, G. (2004). The Nature of Money. Cambridge: Polity.

Keynes, J. (1936). The General Theory of Employment, Interest and Money. London: MacMillan. [Keynes, J. (1978). Teoria generale dell'occupazione, dell'interesse e della moneta e altri scritti. Torino: Bollati]. 
Li Puma, E. \& Lee, B. (2005). Financial Derivatives and the Rise of Circulation. Economy and Society, 34(3), 404- 427.

Luhmann, N. (1988). Die Wirtschaft der Gesellschaft. Frankfurt a.M.: Suhrkamp.

Luhmann, N. (1992). Beobachtungen der Moderne. Opladen: Westdeutscher Verlag.

Luther, M. (1883). Tischreden. En, Werke, vol. 1. Weimar: Hermann Böhlau Nachfolger.

Moore, B. (1979). Monetary Factors. En A. Eichner (Ed.), A Guide to Post-Keynesian Economics (pp. 120-138). London: MacMillan.

Polany, K. (Ed.) (1957). Trade and Market in the Early Empires. Economies in History and Theory. Glencoe, III: The Free Press.

Pryke, M. \& Allen, J. (2000). Monethized Time-Space: Derivatives -Money's 'new imaginary'?. Economy and Society, 29(2), 264- 284.

Robinson, J. (1971). Economic Heresies. Some Old-Fashioned Questions in Economic Theory. London: MacMillan.

Rotman, B. (1987). Signifying Nothing. The Semiotics of Zero. Basingstoke: MacMillan.

Shackle, G. (1972). Epistemics \& Economics. A Critique of Economic Doctrines. Cambridge: Cambridge University Press.

Shackle, G. (1990). Time, Expectations and Uncertainty in Economics. (J. Ford \& E. Elgar, Eds.). Aldershot: Edward Elgar.

Simmel, G. (1889) Zur Psychologie des Geldes. Jahrbuch für Gesetzgebung, Verwaltung und Volkswirtschaft im Deutschen Reich, 13, 1251-1264. [Simmel, G. (1998). II denaro nella cultura modema. Roma: Armando].

Simmel, G. (1900). Philosophie des Geldes. Leipzig: Duncker \& Humblot. [Simmel, G. (1984). Filosofia del denaro. Torino: UTET].

Smithin, J. (Ed.) (2000). What is Money? London: Routledge.

Sohn- Rethel, A. (1990). Das Geld, die bare Münze des Apriori. Berlin: Wagenbach. [SohnRethel, A. (1991). Il denaro. L'aprioriin contanti. Roma: Editori Riuniti]

Voegelin, E. (1925). Die Zeit in der Wirtschaft. Archiv für Sozialwissenschaft und Sozialpolitik, 53(1), 186- 211.

Weber, M. (1922). Wirtschaft und Gesellschaft. Tübingen: J.C.B. Mohr (Paul Siebeck). [Weber, M. (1995). Economia e società. Milano: Edizioni di Comunità].

\section{Sobre la autora}

Elena Esposito es profesora del Departamento de Comunicación y Economía de la Universidad de Módena y Reggio Emilia, Italia. Doctora en Sociología por la Universidad de Bielefeld, Alemania. Entre sus áreas de especialización se encuentran: Sociología de los Medios, Investigación sobre Memoria, Teoría de la Moda y Sociología Económica. Entre sus publicaciones se destacan: Il futuro dei futures. II tempo del denaro nella finanza e nella società [ETS, 2009]; Probabilità improbabili. La realtà della finzione nella società moderna [Meltemi, 2008], I paradossi della moda. Originalità e transitorietà nella società moderna [Baskerville, 2004]; Soziales Vergessen. Formen und Medien der Gedächtnis der Gesellschaft [Suhrkamp, 2002]. En español: (Junto a Giancarlo Corsi y Claudio baraldi) GLU: Glosario sobre la teoría social de Niklas Luhmann [Universidad Iberoamericana, 1996]

\section{Contacto}

Dipartimento di Comunicazione ed Economia Università degli Studi di Modena e Reggio Emilia

viale Allegri 9,

42121 Reggio Emilia

elena.esposito@unimore.it

Recibido: Mayo 2013

Aceptado: Junio 2013 